\title{
Determination of Thermal Plume Thickness using Thermocouple Cross Correlation Coefficient
}

\section{Decay}

\section{Abstract:}

A methodology to measure the thickness of a thermal boundary layer by cross correlating the signal from thermocouples is presented. It is shown that measurement of temperature, velocity, and boundary layer thickness is achievable using a single sensor utilizing three thermocouples rather than the typical two thermocouples used in the cross correlation velocimetry technique discussed in literature. The methodology is validated using experiments performed in two laboratory scale devices: a heated turbulent jet and a variable diameter natural gas burner. Experimental measurements are compared with theoretical calculations, and uncertainty in the measurement is discussed.

Key Words: Cross Correlation Velocimetry, velocity, fire flow, Boundary layer thickness

\section{Introduction:}

Accurate measurements of temperature and velocity fields created by fire plumes are important in quantifying the impact of a fire as well as prediction of ceiling jet velocity, smoke detector and sprinkler activation, requirements for smoke venting systems, and estimation of available safe egress times. Recent large scale experimental studies on fires in buildings have discussed the lack of accurate velocity measurement techniques in fires and the need for research in this area [1].

Quantitative flow measurements in fires are difficult due to the elevated temperatures and caustic environment in fire flows [2]. A velocity probe using the cross correlation of temperature measurements [3] separates itself from other traditional techniques because it is less expensive, robust, and capable of simultaneously measuring temperature and velocity of the flow. This work shows that a modified cross 
correlation velocity probe can also be used to estimate the thermal plume thickness of a fire flow. To measure velocity CCV uses temperature-time records from a set of thermocouples, one downstream of the other, cross-correlated to determine the flow's velocity [3-5]. This technique based on the "frozeneddy" concept in turbulent flows was first proposed by Taylor in 1938 [6]. Taylor hypothesized that in a turbulent flow, there are random and unique eddy structures that retain their shape and characteristics over some time and space. Several studies in fluid mechanics have shown the validity of this assumption [7-9]. Between 1975 and 1980, Cox et al. [3, 10-12] performed a series of experiments that verified the "frozen-eddy" hypothesis in a turbulent flow and showed that velocity measurements could be achieved by means of correlating temperature data from two high response thermocouples. However, due to the high cost associated with analogue correlators available in the 1970's, the technique phased out after the advent of the bidirectional probe which was significantly cheaper at that time. Motevalli [13] and Motevalli et al. $[14,15]$ used the term "Cross Correlation Velocimetry" (CCV) to name the technique. This paper will use the same terminology. CCV has been used more recently in fire related research by Dupuy et al. [16] and Marcelli et al. [17] to measure temperature and upward gas velocity above simulated forest fires. The current study develops a novel approach of CCV towards estimation of a thermal boundary layer thickness. It is shown that the "decay" of cross correlation can be effectively used to measure characteristic thermal boundary layer thicknesses. The methodology is verified using experiments in buoyant heated jets and turbulent gas burner fires.

\section{Operating Principle:}

Figure 1 shows an illustrative sketch of a heated turbulent flow where two thermocouples are used to measure temperature and velocity. 


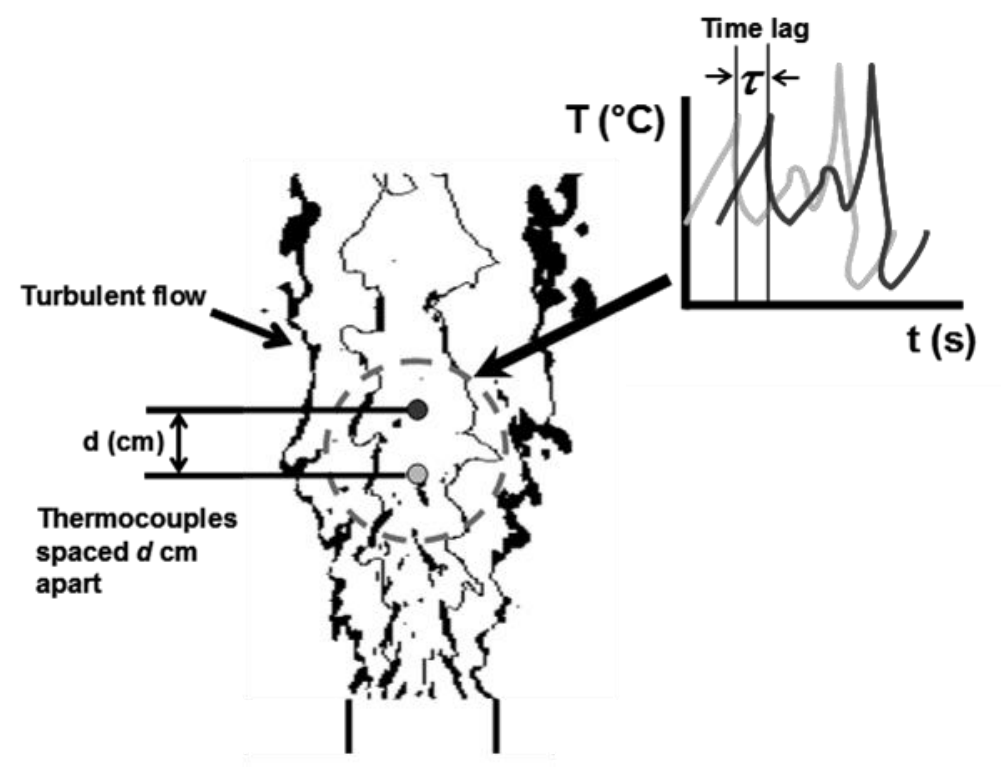

Figure 1: Example of measuring the velocity of a turbulent jet with a CCV probe. Two thermocouples placed $\mathrm{d}(\mathrm{cm})$ apart. A sample temperature profile showing the time lag of the temperature fluctuations is also shown.

The velocity $u(\mathrm{~m} / \mathrm{s})$ of a flow can be calculated using [3],

$$
u=\frac{(d)(f)}{\tau_{s n}},
$$

where $d(\mathrm{~m})$ is the thermocouple separation distance and $\tau_{s n}$ is the nominal sampling lag, or the number of data samples the second signal is delayed behind the first, and $f$ is the sampling frequency which is the same for both thermocouples. $\tau_{s n}$ is found by calculating at what lag the non-dimensional cross coefficient $\rho_{x y}$ has a maximum as shown in Fig. 2. In practice, the thermocouple separation distance and sampling frequency are known quantities. 


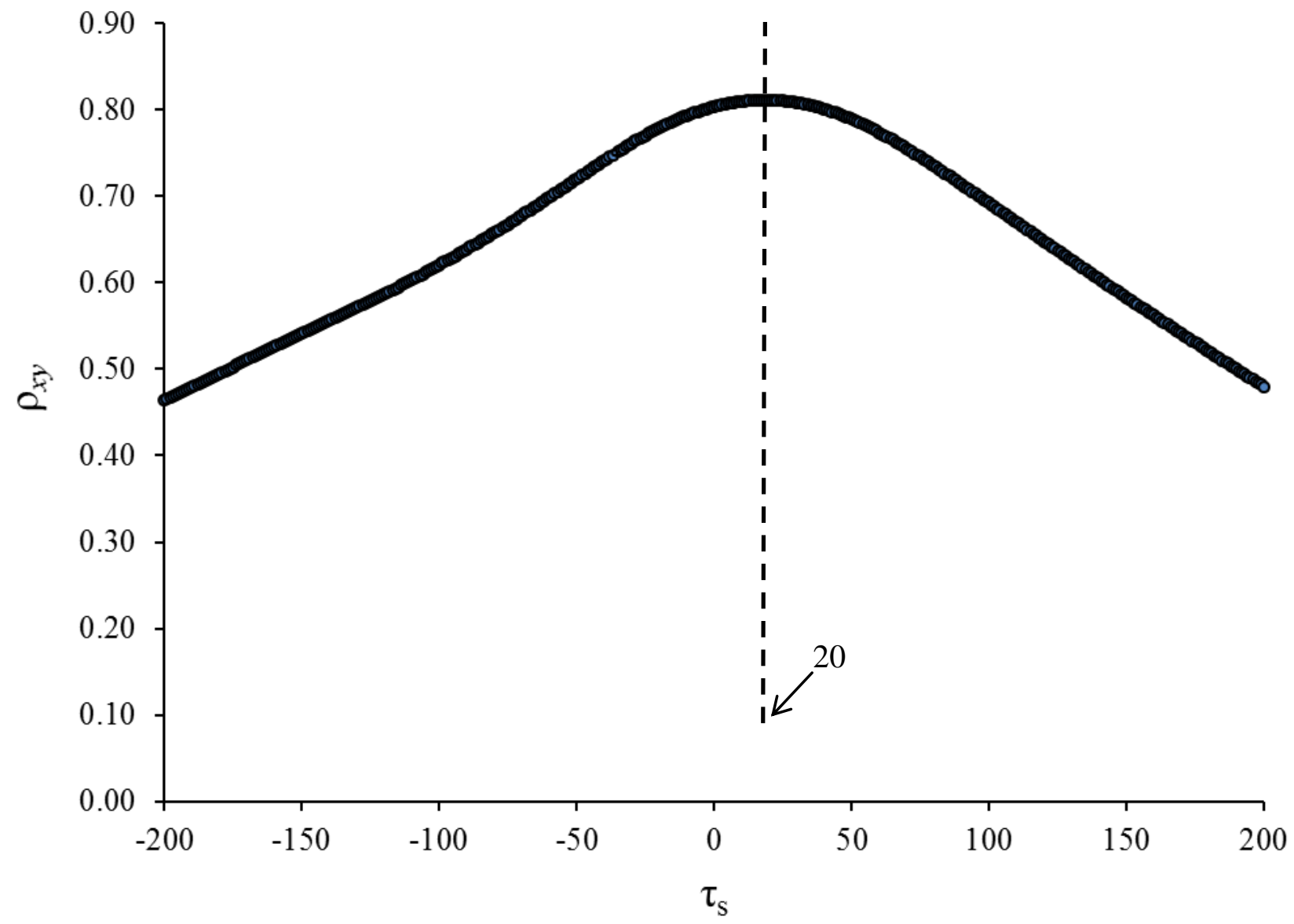

Figure 2: Example of nondimensional cross correlation coefficient $\left(\rho_{\mathrm{xy}}\right)$ verses sampling lag $\left(\tau_{\mathrm{s}}\right)$ using $\boldsymbol{d}=20 \mathrm{~mm}$ and $\boldsymbol{f}=\mathbf{2} \mathrm{kHz}$. (Note: The apparent line displayed is made of closely spaced discrete data points)

The non-dimensionalized cross correlation coefficient $\rho_{x y}$ can be calculated using [12], 


$$
\rho_{x y}=\frac{\lim _{T \rightarrow \infty} \frac{1}{T} \int_{0}^{T} \theta_{x}\left(z-\tau_{s}\right) \theta_{y}(z) d z}{\left[\theta_{x}\left(z-\tau_{s}\right)^{2}\right]^{0.5}\left[\theta_{y}(z)^{2}\right]^{0.5}},
$$

where $\theta=\left(\mathrm{T}_{\mathrm{s}}-\mathrm{T}_{\mathrm{avg}}\right) / \mathrm{T}_{\max } . T_{s}$ is the measured temperature, $T_{\text {avg }}$ is the mean temperature of the data set, $T_{\max }$ is the maximum temperature in the data set, $z$ is an indices location in the $\theta$ profile, $\tau_{s}$ is the sampling lag or the number of samples $\theta_{x}$ is separated from $\theta_{y}, T$ is the total number of samples, and $\theta_{x}(z)$ and $\theta_{y}(z)$ represent the normalized first and second temperature readings respectively. By plotting the cross correlation coefficient $\left(\rho_{x y}\right)$ versus $\tau_{s}$ the nominal sampling lag $\tau_{s n}$ is found as the abscissa of the peak.

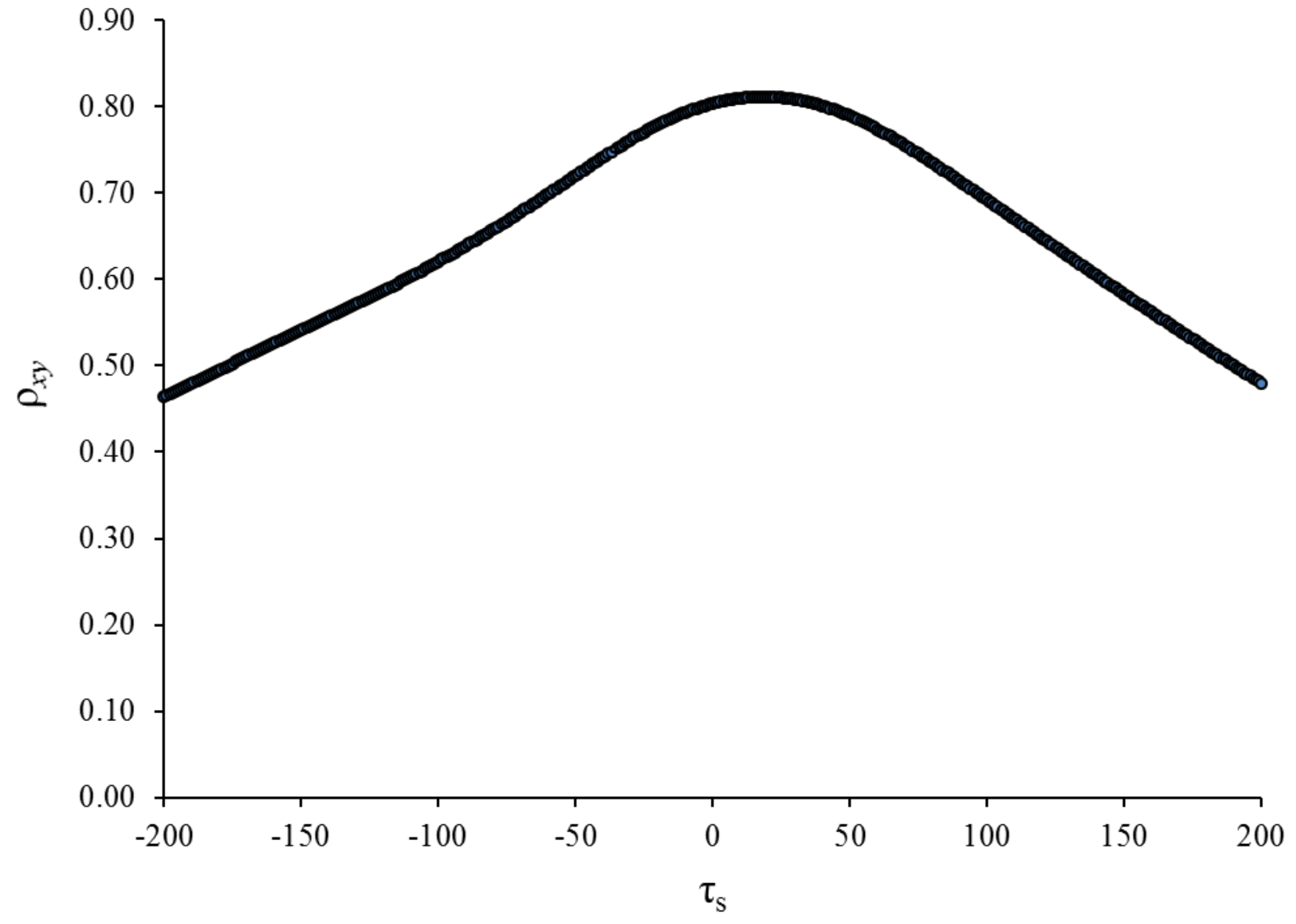

Figure 2 shows an example of $\rho_{x y}$ verses $\tau_{s}$ plot using an $f$ of $2 \mathrm{kHz}$ and a $d$ of $20 \mathrm{~mm}$ where $\tau_{s n}$ is 20 which corresponds to a velocity of $2 \mathrm{~m} / \mathrm{s}$ using Eq. (1).

Signals with a strong correlation have a $\rho_{x y}$ close to unity while signals with a weak correlation have lower values of $\rho_{x y}$. Previous studies $[13,15]$ have shown that $\rho_{x y} \geq 0.5$ is required for a velocity 
measurement. The $\rho_{x y}$ decays due to the loss of the static temperature profile in the turbulent eddy as it moves along the flow path. As the turbulent eddy moves, the gasses continuously rotate and mix which disrupts any recognizable pattern that can be measured. Eventually, a turbulent eddy will change enough that no recognizable temperature profile will be able to be matched with an earlier measurement. Research has shown that a turbulent eddy will potentially exist along a distance equal to the eddy's characteristic turbulent length scale [19]. Based on the required cross correlation coefficient and the turbulent length scale information and if the decay in the cross correlation coefficient is predictable, an estimation of the characteristic length scale can be determined using a measurement of the decay in the $\rho_{x y}$ and extrapolating it to the cutoff point of $\rho_{x y}$. This is done in two separate experiments as discussed below.

\section{Experimental Setup:}

Two experiments were performed to analyze the relationship between the decay in the cross correlation coefficient and the thermal plume thickness of a heated turbulent flow. The first is an axisymmetric jet, and the second is a gas burner.

\subsection{Heated turbulent jet experiment}

Figure 3 shows the axi-symmetric jet experimental setup used in this study. The jet diameter was equal to $5 \mathrm{~cm}$ and measurements were taken $46 \mathrm{~cm}$ above the nozzle, beyond the potential core which can extend 4-6 nozzle diameters $(20-30 \mathrm{~cm})$ into the jet [18]. An electric fan pushed air over electric heaters to generate a heated flow with a temperature between 2 to $20{ }^{\circ} \mathrm{C}$ above ambient, measured at the nozzle exit. The flow velocity ranged from $0.1 \mathrm{~m} / \mathrm{s}$ to $2 \mathrm{~m} / \mathrm{s}$. The jet (nozzle diameter $=5 \mathrm{~cm}$ ) expands into a clear Plexiglas cage, with dimensions of 46 x 46 x $122 \mathrm{~cm}$. Two E-type thermocouples, with wire sizes of $8 \times 10^{-5} \mathrm{~m}(0.003$ inches $)$ were used to make the temperature measurements. E-type thermocouples 
were used because they have a relatively large voltage output $\left(61 \mu \mathrm{V} /{ }^{\circ} \mathrm{C}\right)$ compared to other commercially available thermocouples such as K-type $\left(40 \mu \mathrm{V} /{ }^{\circ} \mathrm{C}\right)$. The two thermocouples were inserted in two hollow ceramic tubes (diameter $=3.175 \mathrm{~mm}$ and $1.587 \mathrm{~mm}$ ). The thermocouple separation distance can be adjusted between 5 to $120 \mathrm{~cm}$ in the vertical direction.

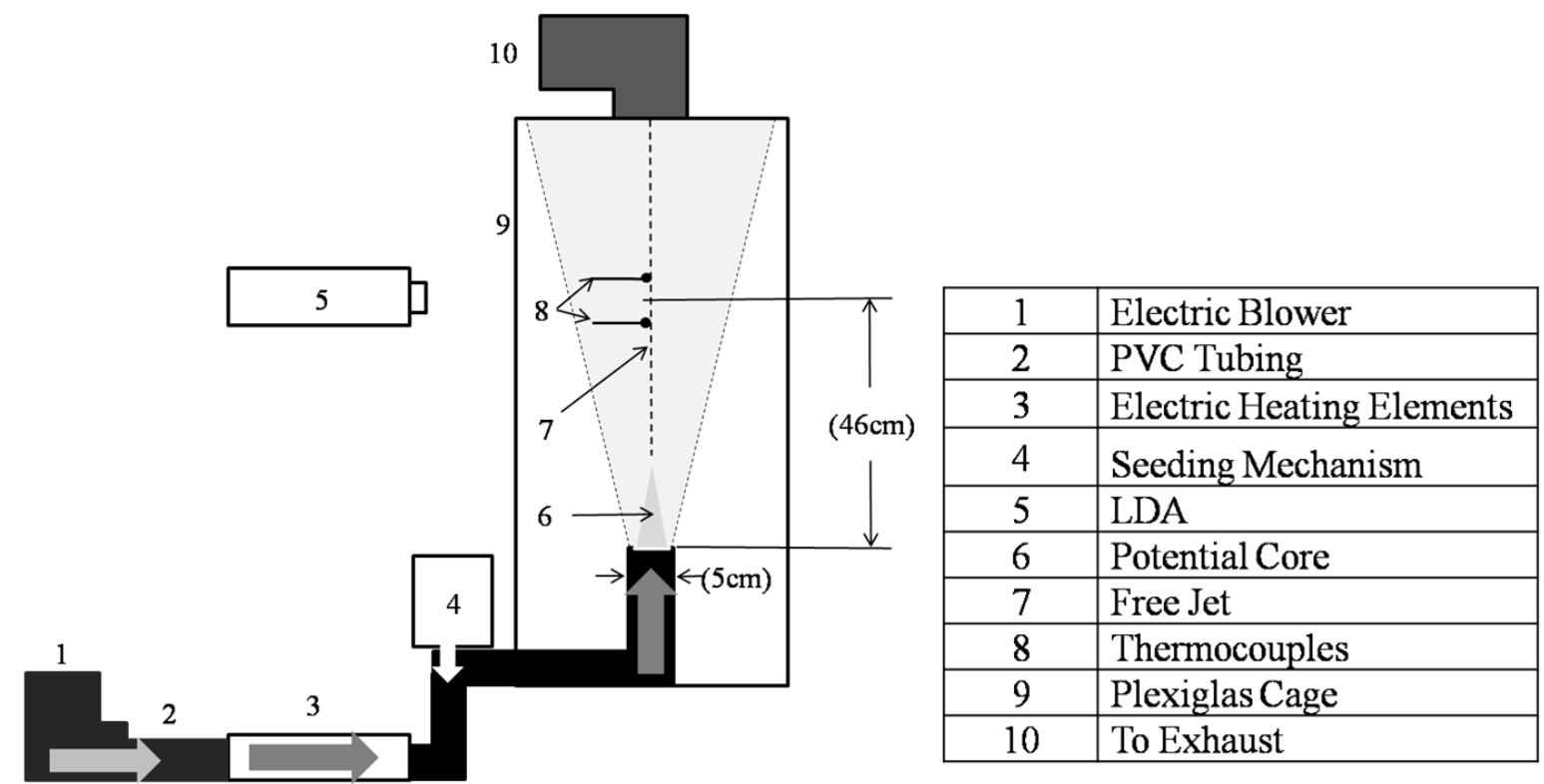

Figure 3: Diagram of Axi-symmetric jet experiment.

To confirm that the thermocouples had similar response times, the probes were reversed in a constant flow, and comparable results were obtained. The separation distance between thermocouples could be varied with accuracy up to $\pm 0.01 \mathrm{~mm}$ in the vertical plane while keeping the measurement volume at the same height above the jet nozzle. In the vertical plane the thermocouples were aligned using a laserbased alignment system which decreased the error due to misalignment. An Intelligent Laser Applications $75 \mathrm{~mW}$ fixed optical path length fp50-shift Laser Doppler Anemometer (LDA) system with an accuracy of $\pm 0.5 \%$ of the measured velocity was used to calibrate the flow velocity for each experiment. Flow seeding was achieved using $12 \mu \mathrm{m}$ zirconium dioxide particles. A Honeywell Model 
16200 hepa filter was used to remove the seed particles from the flow after the heated air passed out of the measurement volume.

\subsection{Turbulent gas burner experiment}

In addition to an axi-symmetric jet, a natural gas burner was also built as shown in Fig. 4. The burner consists of a $1.22 \mathrm{~m}$ by $1.22 \mathrm{~m}$ square drywall top with the burner in the centre. The diameter of the burner was adjusted by attaching a steel plate with a hole equal to the desired burner size. Natural gas was piped through a $2.54 \mathrm{~cm}$ stainless steel braided tube into an air gap created by wire mesh, $5 \mathrm{~cm}$ of Kaowool and $10 \mathrm{~cm}$ of sand in order to provide an even distribution of gas over the burner surface. A three thermocouple $\mathrm{CCV}$ probe was used so that the separation distance did not have to be changed manually over the flames. The thermocouple separation distances were adapted from the axi-symmetric

jet experiments and set at 4, 8 , and $12 \mathrm{~cm}$ providing 3 separation distances with which to calculate the $\rho_{x y}$ decay. Fires having base diameters of $10 \mathrm{~cm}, 15 \mathrm{~cm}$, and $20 \mathrm{~cm}$ and heat release rates between 6.2 $\mathrm{kW}$ to $23.7 \mathrm{~kW}$ were tested. The heat release rate was determined by adjusting the flow of natural gas to the burner. Measurements were taken at 4 heights above the plume $(0.65 \mathrm{~m} 0.98 \mathrm{~m}, 1.22 \mathrm{~m}, 1.54 \mathrm{~m})$. The plume width was measured using a horizontal thermocouple tree using eight $\mathrm{E}$ type thermocouples with wire diameters between $0.8128 \mathrm{~mm}$ and $0.254 \mathrm{~mm}$. 


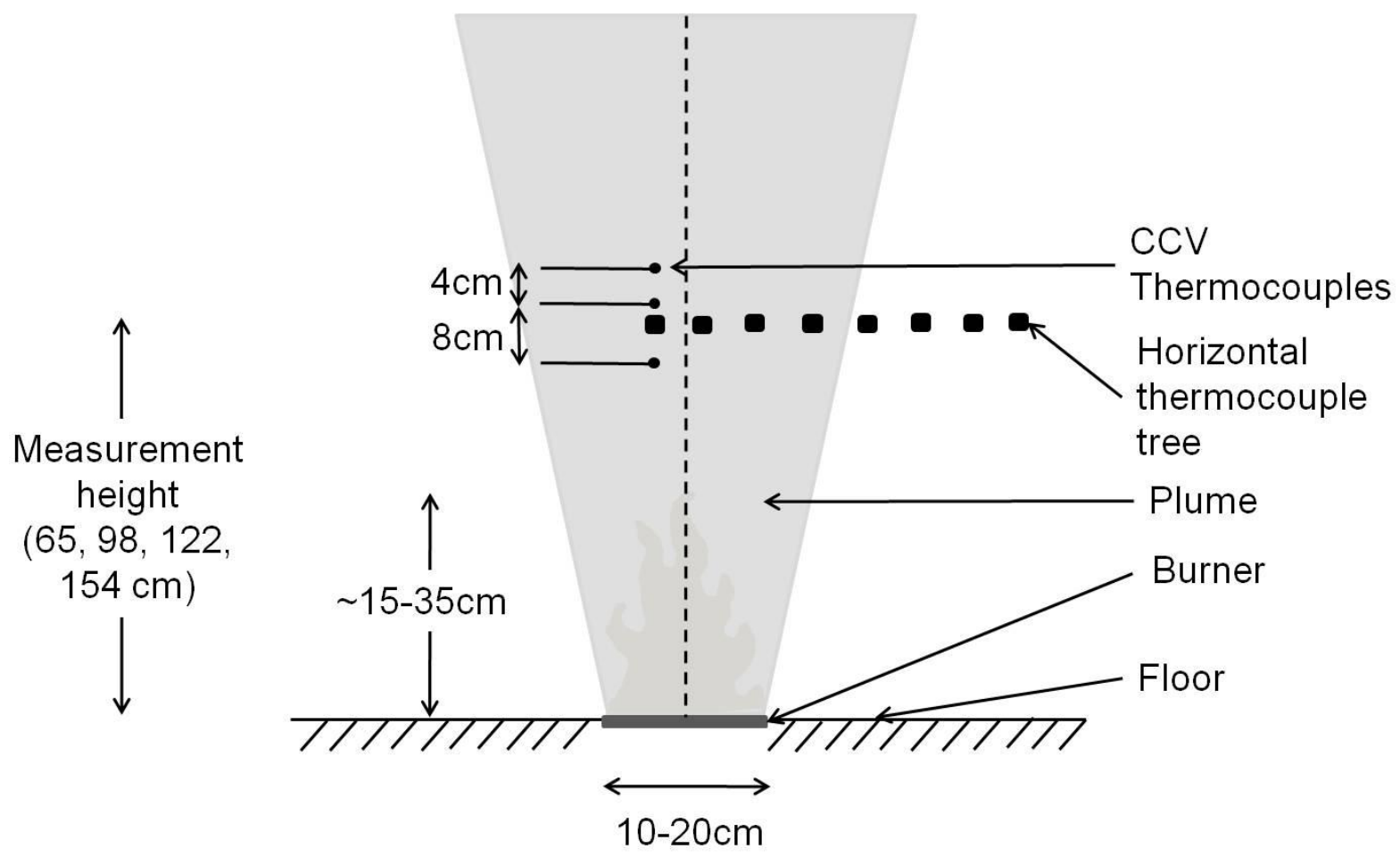

Figure 4: Diagram of axi-symmetric burner experiment.

\section{Results and Analysis:}

Figure 5 shows the decay in the cross correlation $\left(\rho_{x y}\right)$ as a function of the separation distance between the two thermocouples placed along the vertical axis of a heated turbulent jet (Fig. 3). Interestingly, $\rho_{x y}$ decays linearly as shown in Fig. 5. Extrapolating the decay to $\rho_{x y} \sim 0.5$ as shown in Fig.5 gives a separation distance of $197 \mathrm{~mm}$ which is in close agreement to the thermal plume width at this location. As discussed earlier, $\rho_{x y} \sim 0.5$ corresponds to a value where, statistically, the correlation between two signals is unrecognizable. The thermal plume width for a circular jet can be calculated from standard heat transfer textbooks and is given by [18]: 


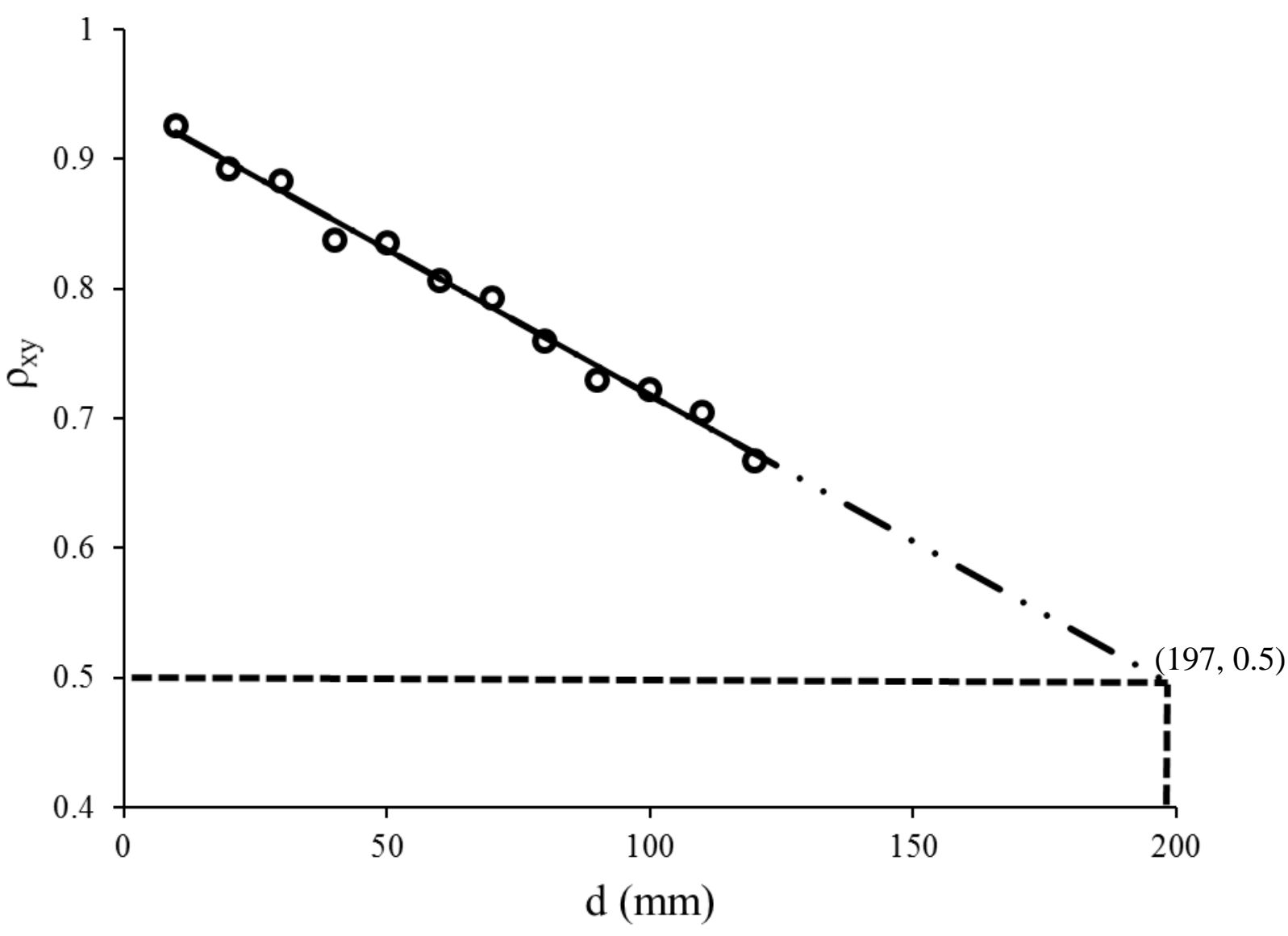

Figure 5: Nondimensional cross correlation coefficient $\left(\rho_{\mathrm{xy}}\right)$ versus thermocouple separation distance $(d)$ using experimintal paremeters of: $f=2 \mathrm{kHz}, t_{T}=15 \mathrm{~s}$, and $\mathrm{u}=1.5 \mathrm{~m} / \mathrm{s}$.

$$
\frac{2 \delta}{d_{i}}=\left[1+24 C \frac{x}{d_{i}}\right],
$$

where $\delta$ is the jet radius, $d_{i}$ is the nozzle diameter $(5 \mathrm{~cm}), x$ is the height above the nozzle $(46 \mathrm{~cm})$, and $C$ is an empirical constant equal to 0.0128 [18]. Using Eq. (3) the width of the jet at the measurement location was calculated as $191 \mathrm{~mm}$, which is reasonably close to the $197 \mathrm{~mm}$ predicted by CCV 
technique. These values are within a $3 \%$ level of uncertainty $(197+/-6[\mathrm{~mm}])$ but this single data point is not enough to determine a the accuracy of the technique as a single sensor so more experimentation was conducted.

To further analyse the use of the decay in $\rho_{x y}$ to estimate the width of a flow, CCV measurements with varying separation distances were taken along the centreline of the flow, and velocity measurements were taken with the LDA along the radius of the jet at different heights above the nozzle. The CCV measurements were extrapolated to find the separation distance where the $\rho_{\mathrm{xy}}$ decays to 0.5 . The edge of the jet was defined as a region when velocity decayed to $85 \%$ of the maximum (velocity along centreline). Experimental results of the CCV measurements, the LDA measurements, and Eq. (3) are compared in Fig. 6 showing good agreement. Figure 6 also clearly demonstrates the validity of the concept of using the decay in cross correlation towards measurement of the thermal boundary layer in a heated non combusting jet. These experimental measurements are within a $15 \%$ level of uncertainty (error bars of $15 \%$ of $\mathrm{CCV}$ measurements value are shown on Figure 6) of the theoretical calculation of Eq. (3). This represents the maximum in these experiments. The increase in uncertainty is likely due to using 3 thermocouple separation distances rather than the twelve shown in figure 5 to make the extrapolation $\rho_{x y}=0.5$. The CCV measurements have a much smaller maximum uncertainty $(7 \%)$ when compared to the LDA measurements of the flow rather than the theoretical value of Eq. (3). 


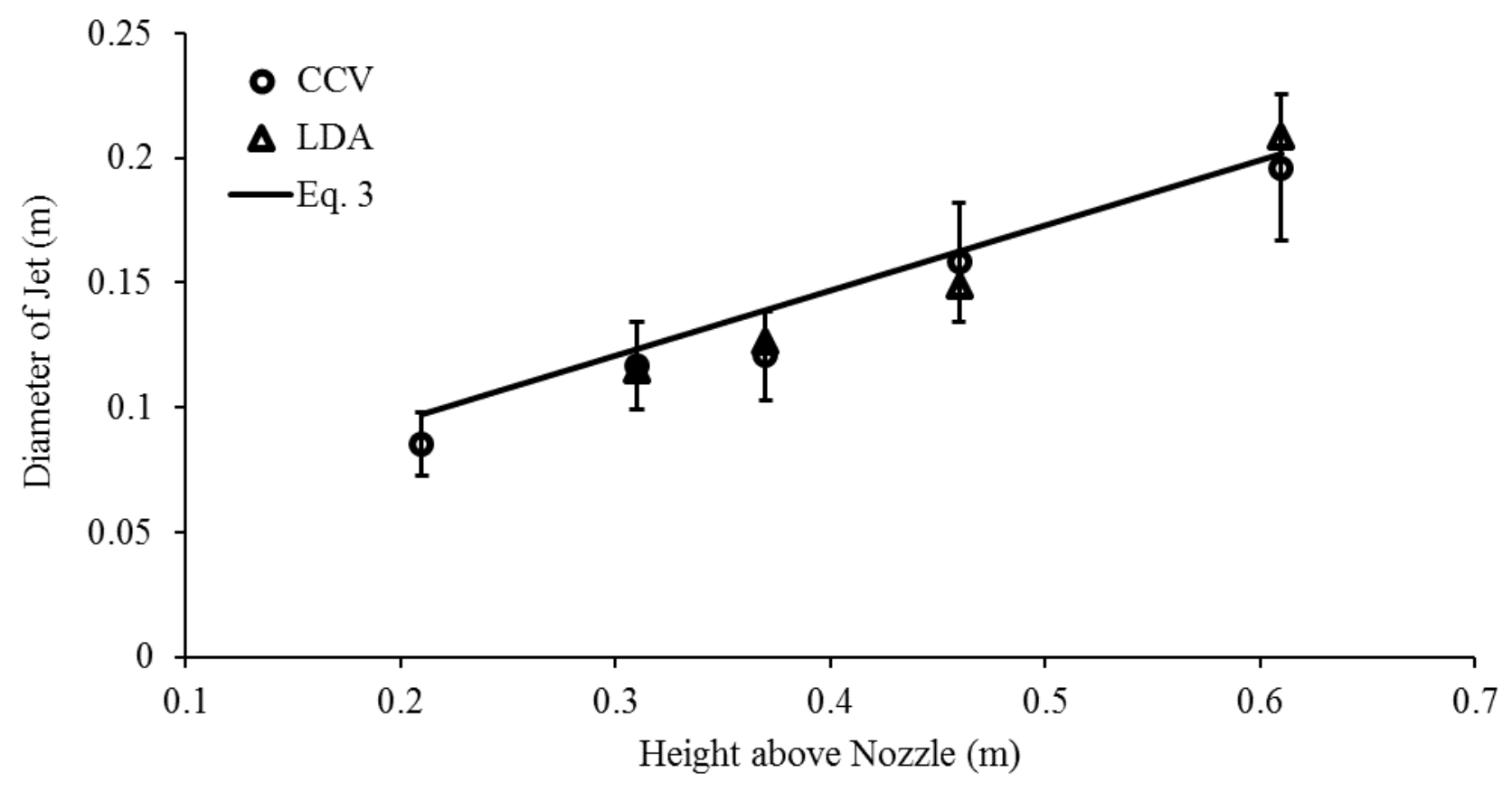

Figure 6: Comparison of measured and theoretical diameter of turbulent free jet versus height above nozzle.

Figure 7 shows the plume width obtained using the temperature profile versus the width predicted by the decay in $\rho_{x y}$ for three burner diameters and six different heat release rates. To find the point of $85 \%$ decay in the temperature profile, the recorded data from 8 thermocouples was fitted to a fifth order polynomial. Figure 7 shows that the plume widths measured using CCV were $8.4 \%$ lower than the thermocouple width measurements. To expand on the statistical uncertainty discussion, the majority of these experimental measurements are within a $20 \%$ level of uncertainty (error bars of $20 \%$ of CCV measurements value are shown on Figure 7). This increase in uncertainty over the axi-symmetric jet experiment is likely due to the lack of conditional control available in an open burner setup. Potential 
sources of variation include an offset in the alignment of the thermocouples along the centerline of the plume and varying plume angles due to ambient airflow in the laboratory fume hood. However, the results are promising as they allow measurement of combusting turbulent plumes using an array of three thermocouples traversed along the vertical axis of the burner. A single 3 thermocouple CCV sensor can be used to measure temperature, velocity, and the thickness of a heated turbulent plume.

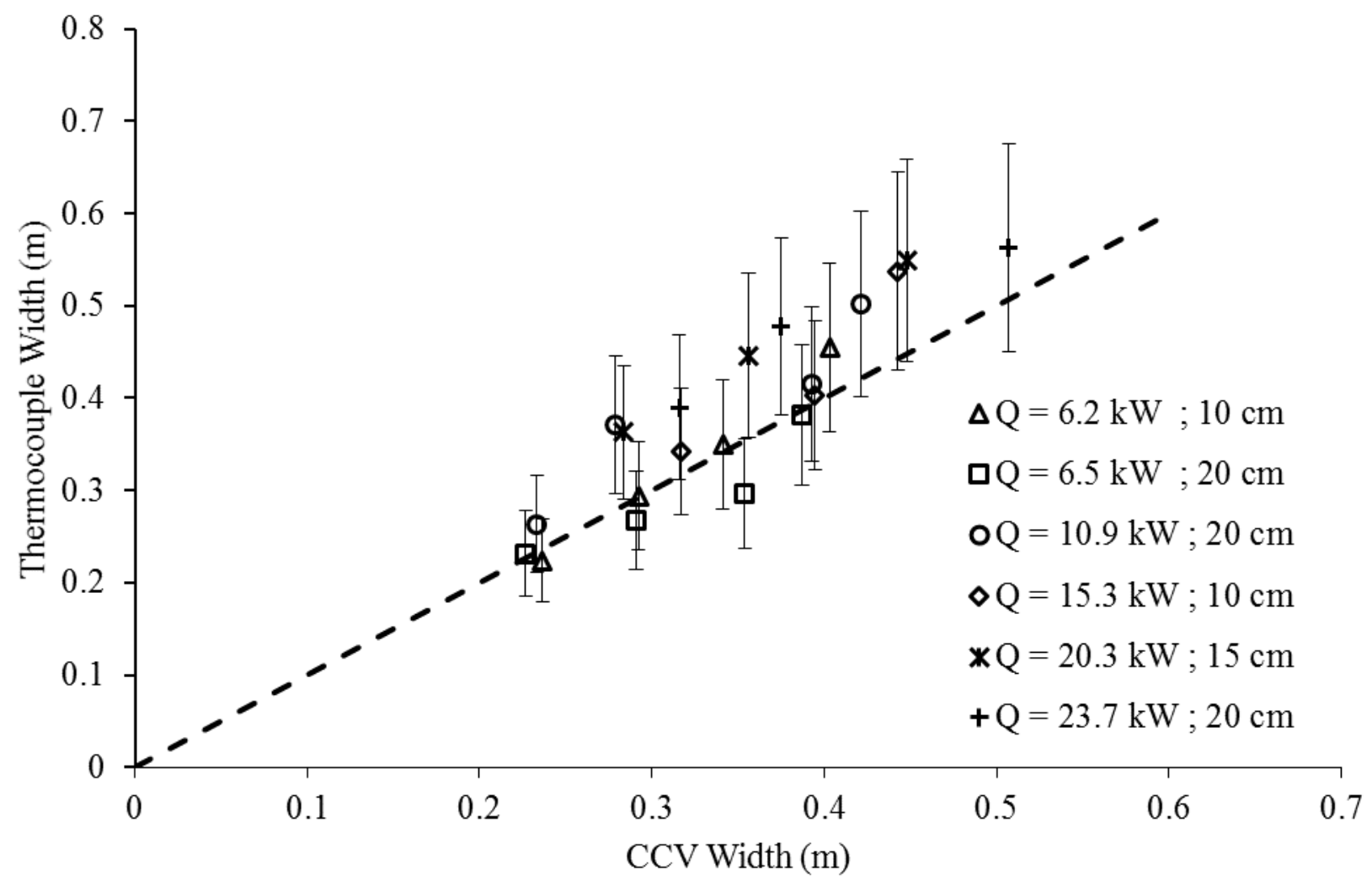

Figure 7: Plume width measured using a thermocouple tree versus plume width predicted by the decay in the CCV nondimensional cross correlation coefficient. $f=10 \mathrm{kHz}, \mathrm{t}_{\mathrm{T}}=10 \mathrm{~s}$. 


\section{Conclusions:}

A novel technology to measure the thickness of a thermal boundary layer by cross correlating the signal from thermocouples is presented. Experimental results using a heated, turbulent, axisymmetric jet show that the cross correlation coefficient decays linearly as a function of distance between two thermocouples and the measured rate of decay can be correlated to the width of the thermal boundary layer. Comparison with Laser Doppler Anemometer (LDA) measurements show good agreement with the proposed technology. The novel technique was also tested with success using a gas burner showing that boundary layer widths of chemically reacting buoyancy driven flows with natural mixing and turbulence generation can also be measured using this technique The advantages of the proposed technique compared to existing methods are the simplicity in design, low cost, and accuracy comparable to existing methods such as LDA. Further, a single probe with three thermocouples can make temperature, velocity, and boundary layer thickness measurements of heated turbulent flows.

\section{Acknowledgements:}

The author would also like to thank the Society of Fire Protection Engineering, and the National Science Foundation Graduate Research Fellowship Program for helping fund this research.

\section{References:}

1. Torero, J.L. and Carvel, R., The dalmarnock fire tests: Experiments and modeling, ed. G. Rein, C.A. Empis, and R. Carvel. 2007: School of Engineering and Electronics, University of Edinburgh.

2. Tieszen, S.R., On the fluid mechanics of fires. Annual Rev. Fluid Mech., 2001. 33: p. 67-92.

3. Cox, G., Gas velocity measurement in fires by the cross correlation of random thermal fluctuations, in Fire Research Note 10321975: Borehamwood. 
4. Marvasti, M.A. and Strahle, W.C., Fractal interpolation methods in spatial cross-correlation velocimetry. Experiments in Fluids, 1994. 18: p. 129-130.

5. Morgan, M.G. and Bowles, K.L., Cross-correlation and cross-spectral methods for drift velocity measurements. Science, 1968. 161(3846): p. 1139-1142.

6. Taylor, G.I., The spectrum of turbulence. Proc Roy. Soc. A, 1938. 164: p. 476-490.

7. Antonia, R.A., Chambers, A.J., and Thein, N.P., Taylor's hypothesis and spectra of velocity and temperature derivatives in a turbulent shear flow. Boundary-Layer meterology, 1979. 19(1): p. $19-29$.

8. Burghelea, T., Segre, E., and Steinberg, V., Validity of the taylor hypothesis in a random spatially smooth flow. Physics of Fluids, 2005. 17.

9. Cenedese, A., Romano, G.P., and Felice, F.D., Experimental testing of taylor's hypothesis by lda in highly turbulent flow. Experiments in Fluids, 1991. 11: p. 351-358.

10. Cox, G., Some measurements of fire turbulence. Fire and Mat., 1976. 1: p. 116-122.

11. Cox, G. and Chitty, R., Study of the deterministic properties of unbounded fire plumes. Comb. and Flame, 1980. 39: p. 191-209.

12. Cox, G., Gas velocity measurements in fires by the cros-correlation of random thermal fluctuations- a comparison with conventional techniques. Comb. and Flame, 1977. 28: p. 155163.

13. Motevalli, V., A study of the characteristics of small-scale unconfined fire induced ceiling jets, in ME1989, University of Maryland, College Park.

14. Motevalli, V. and Marks, C.H., Transient and steady state study of small-scale, fire-induced unconfined ceiling jets, in AIAA/ASME Thermophysics and Heat Transfer Conference1990, HTD. p. 46-61. 
15. Motevalli, V., Marks, C.H., and Mccaffrey, B.J., Cross-correlation velocimetry for measurement of velocity and temperature profiles in low-speed, turbulent, nonisothermal flows. Journal of Heat Transfer (Transactions of the ASME), 1992.

16. Dupuy, J.L., Marechal, J., and Morvan, D., Fires from a cylindrical forest fuel burner: Combustion dynamics and flame properties. Comb. and Flame, 2003. 135(1-2): p. 65-76.

17. Marcelli, T., Santoni, P.A., Simeoni, A., Leoni, E., and Porterie, B., Fire spread across pine needle fuel beds: Characterization of temperature and velocity distributions within the fire plume. International Journal of Wildland Fire, 2004. 13: p. 37-48.

18. Kanury, A.M., An introduction to combustion phenomena. 1977: Gordon \& Breach Science Publishers, Inc.

19. Coats, C.M., Coherent structures in combustion. Prog. Energy Combustion Science, 1996. 22: p. 427-509.

20. Karlsson, B. and Quintiere, J.G., Enclosure fire dynamics. 2000: CRC Press.

21. Hirano, T. and Saito, K., Fire spread phenomena: The role of observation in experiment. Progress in Energy and Combustion Science, 1994. 20(6): p. 461-485. 